\title{
Optimal shapes for anguilliform swimmers at intermediate Reynolds numbers
}

\section{Journal Article}

\section{Author(s):}

Rees, Wim M. van; Gazzola, Mattia; Koumoutsakos, Petros

Publication date:

2013-05

Permanent link:

https://doi.org/10.3929/ethz-b-000067815

\section{Rights / license:}

In Copyright - Non-Commercial Use Permitted

\section{Originally published in:}

Journal of Fluid Mechanics 722, https://doi.org/10.1017/jfm.2013.157 


\title{
JFM RAPIDS journals.cambridge.org/rapids
}

\section{Optimal shapes for anguilliform swimmers at intermediate Reynolds numbers}

\author{
Wim M. van Rees, Mattia Gazzola and Petros Koumoutsakos $\dagger$ \\ Computational Science and Engineering Laboratory, Clausiusstrasse 33, \\ ETH Zürich, CH-8092, Switzerland \\ (Received 18 January 2013; revised 8 March 2013; accepted 13 March 2013; \\ first published online 8 April 2013)
}

We investigate the optimal morphologies for fast and efficient anguilliform swimmers at intermediate Reynolds numbers, by combining an evolution strategy with threedimensional viscous vortex methods. We show that anguilliform swimmer shapes enable the trapping and subsequent acceleration of regions of fluid transported along the entire body by the midline travelling wave. A sensitivity analysis of the optimal morphological traits identifies that the width thickness in the anterior of the body and the height of the caudal fin are critical factors for both speed and efficiency. The fastest swimmer without a caudal fin, however, still retains $80 \%$ of its speed, showing that the entire body is used to generate thrust. The optimal shapes share several features with naturally occurring morphologies, but their overall appearances differ. This demonstrates that engineered swimmers can outperform biomimetic swimmers for the criteria considered here.

Key words: biological fluid dynamics, propulsion, swimming/flying

\section{Introduction}

The striking hydrodynamic performance of natural swimmers (Hertel 1966) has inspired a wide range of engineering applications, from robots to energy-harnessing systems (Triantafyllou \& Triantafyllou 1995; Nachtigall \& Bluechel 2000; Bartol et al. 2005). Bioinspired engineering designs often rely on mimicking specific features of natural organisms that are considered to have a functional role (Wilga \& Lauder 2004; Nawroth et al. 2012).

In the case of natural swimmers, however, we have limited information to assess which functions are served by their morphological features, and whether such shapes provide an optimal solution. Furthermore, engineering designs may take advantage of available materials and components with flexibilities and restrictions different from

\section{$\dagger$ Email address for correspondence: petros@ethz.ch}




\section{W. M. van Rees, M. Gazzola and P. Koumoutsakos}

those encountered in natural organisms. One may therefore question to what extent engineers should follow a biomimicry approach. In this context the work of Borazjani \& Sotiropoulos (2010) demonstrated that manually selected combinations of different shapes and kinematics can swim faster than naturally occurring swimmers. In Gazzola, van Rees \& Koumoutsakos (2012) it was found that C-start escape mechanisms of larval fish can be improved upon by increasing the midline curvature beyond values observed in nature. In the case of potential flow models, optimal fast and efficient shapes and motions have been identified through multi-objective optimization (Tokic \& Yue 2012; Eloy 2013).

Here we present shape optimizations with regard to speed and efficiency for selfpropelled anguilliform swimmers at moderate Reynolds numbers. We analyse the geometric features that lead to optimality and discuss how they relate to corresponding biological systems.

The paper is organized as follows. The shape parametrization and cost functions are presented in $\S 2$, followed by a brief review of the employed methodology in $\S 3$. The results of the optimization campaigns are analysed in $\$ \S 4$ and 5 , and the findings are summarized in $\S 6$.

\section{Physical problem definition}

\subsection{Flow conditions}

In this work we study swimmers at a constant Reynolds number based on the fish length $L$, period of the travelling wave $\mathscr{T}$ and dynamic viscosity $v, R e_{\text {fish }}=$ $\left(L^{2} / \mathscr{T}\right) / v=550$ (Gazzola et al. 2012). This flow regime is typical for larval zebrafish 5 days post-fertilization (Muller, van den Boogaart \& van Leeuwen 2008; Gazzola et al. 2012), which will be used here as the biological reference to place the optimal solutions into context.

\subsection{Midline kinematics}

The swimmer's midline kinematics (see the Appendix in the supplementary material available at http://dx.doi.org/10.1017/jfm.2013.157) is fixed throughout this work to a motion representative of anguilliform swimming (Carling, Williams \& Bowtell 1998). Anguilliform swimming is an archetypal swimming mode that has been extensively studied, both experimentally (Gray 1933; Tytell 2004) and computationally (Carling et al. 1998; Kern \& Koumoutsakos 2006). Furthermore, the larval zebrafish considered here as reference exhibits anguilliform swimming motion (Muller et al. 2008).

\subsection{Shape parametrization}

The three-dimensional geometry of the swimmer is represented by two orthogonal planar curves for the width and height profiles, respectively. Each curve is a bijective, continuous and smooth function of the axial coordinate $s$. For each $s \in[0, L]$, an elliptical cross-section is defined by half-axes based on the width and height curves $w(s)$ and $h(s)$, respectively. The union of cross-sections is the employed doubly symmetric three-dimensional geometry. We note that, by fixing the length to keep $R e_{\text {fish }}$ constant, the volume varies for different shapes.

The height and width profiles are represented by cubic B-splines with $N_{h}$ and $N_{w}$ control points (figure 1). For both curves the first and last control points are fixed at $\left(s_{0}^{w}, w_{0}\right)=\left(s_{0}^{h}, h_{0}\right)=(0,0)$ and $\left(s_{N_{w}-1}^{w}, w_{N_{w}-1}\right)=\left(s_{N_{h}-1}^{h}, h_{N_{h}-1}\right)=(L, 0)$, respectively, and serve to maintain $C^{1}$ continuity at the end points. The remaining control points are uniformly distributed along the length of the swimmer. Their width and 
(a)

$w / L$

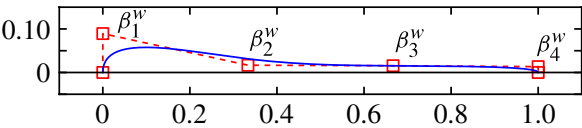

$h / L$

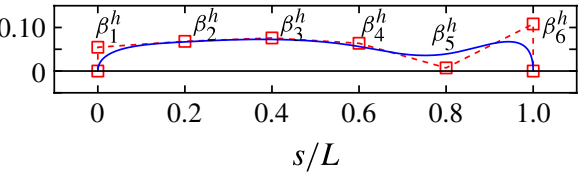

(b)

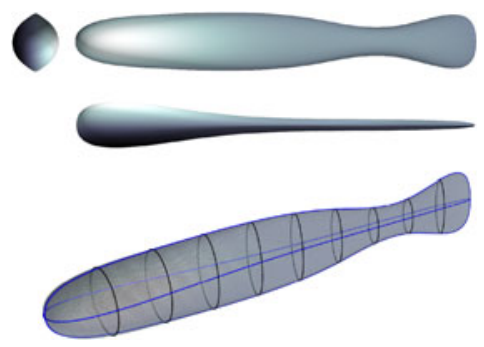

FIGURE 1. Three-dimensional shape reconstruction of a larval zebrafish 5 days postfertilization $\left(\boldsymbol{m}_{0}\right)$ based on our parametrization. (a) The width and height profiles (solid blue curves) are cubic B-splines defined by the parameter values $\beta_{i}^{w}$ and $\beta_{j}^{h}$, respectively. $(b)$ The three-dimensional shape is generated by constructing the ellipsoidal cross-sections with halfaxes defined by the width and height profiles.

height values, $\beta_{i}^{w}$ and $\beta_{j}^{h}$ with $i \in\left[1, N_{w}-2\right]$ and $j \in\left[1, N_{h}-2\right]$, are the parameters to be optimized. We set $N_{w}=6$ and $N_{h}=8$, for a total of ten parameters, $\left\{\beta_{i}^{w}\right\}_{i=1, \ldots, 4}$ and $\left\{\beta_{j}^{h}\right\}_{j=1, \ldots, 6}$. Example shapes generated with this parametrization are shown in the Appendix in the supplementary material.

We remark that in the present simulations the swimming motion is imposed irrespective of the power required to sustain it. Swimmers with large vertical and lateral dimensions will exert correspondingly large forces on the fluid to achieve the prescribed motion. In order to facilitate a comparison with natural swimmers and to generate shapes that are relevant for robotic design, we constrain the search space so that the swimmer fits within a bounding box of size $L \times 0.3 L \times 0.3 L$. This size of the bounding box is chosen so as to allow a wide range of shapes while retaining relevance to natural swimmers and man-made vehicles.

\subsection{Cost function for fast swimming}

The fastest shape is found by maximizing the average forward velocity $\bar{U}$ over the sixth swimming cycle, starting from rest. The sixth cycle was chosen as a compromise between reducing computational costs and approximating cruise velocity, based on preliminary studies and the results of Kern \& Koumoutsakos (2006). The cost function to be minimized during the optimization is defined as

$$
f_{v e l}=-\bar{U}=-\left\|\frac{1}{\mathscr{T}} \int_{5 \mathscr{T}}^{6 \mathscr{T}} \boldsymbol{U}(t) \mathrm{d} t\right\|_{2},
$$

where $\boldsymbol{U}(t)$ is the velocity vector of the swimmer's centre of mass.

\subsection{Cost function for efficient swimming}

The most efficient shape is identified by maximizing, over the sixth cycle, the Froude efficiency, i.e. the ratio between useful energy and the sum of input and useful energy. The input energy is imparted by the swimmer to the fluid through its deformation while the useful energy is the kinetic energy gained by the swimmer itself. The 
efficiency cost function is defined as

$$
f_{\text {eff }}=-\frac{E_{\text {useful }}}{E_{\text {input }}+E_{\text {useful }}}=-\frac{m \bar{U}^{2} / 2}{\left(\frac{1}{\mathscr{T}} \int_{5 \mathscr{T}}^{6 \mathscr{T}} P_{\text {input }}(t) \mathrm{d} t\right)+m \bar{U}^{2} / 2},
$$

where $m$ is the swimmer's mass and $P_{\text {input }}$ is the total instantaneous power delivered to the fluid (see the Appendix in the supplementary material). The swimmer's volume is part of the efficiency definition, which can therefore be understood from an engineering perspective as a transport efficiency.

We stress that our objective is to identify optimal swimmer morphologies with respect to relevant engineering metrics. Natural fish have probably evolved under requirements and constraints different from those considered in this study.

\section{Methodology}

\subsection{Numerical method}

We consider a self-propelling body immersed in a three-dimensional viscous flow governed by the incompressible Navier-Stokes equations:

$$
\boldsymbol{\nabla} \cdot \boldsymbol{u}=0, \quad \frac{\partial \boldsymbol{u}}{\partial t}+(\boldsymbol{u} \cdot \nabla) \boldsymbol{u}=-\frac{1}{\rho} \nabla p+v \nabla^{2} \boldsymbol{u}, \quad \boldsymbol{x} \in \Sigma \backslash \Omega,
$$

where $\Sigma$ is the entire domain and $\Omega$ is the volume occupied by the swimmer. The no-slip boundary condition at the geometry interface $\partial \Omega$ matches the fluid velocity $\boldsymbol{u}$ to the local body velocity $\boldsymbol{u}_{s}$. The feedback from the fluid to the body follows Newton's equations of motion:

$$
m_{s} \ddot{\boldsymbol{x}}_{s}=\boldsymbol{F}^{H}, \quad \mathrm{~d}\left(\boldsymbol{I}_{s} \dot{\boldsymbol{\theta}}_{s}\right) / \mathrm{d} t=\boldsymbol{M}^{H},
$$

where $\boldsymbol{F}^{H}$ and $\boldsymbol{M}^{H}$ are the hydrodynamic force and momentum exerted by the fluid on the body, characterized by centre of mass $\boldsymbol{x}_{s}$, angular velocity $\dot{\boldsymbol{\theta}}_{s}$, mass $m_{s}$ and moment of inertia $\boldsymbol{I}_{s}$.

The numerical method to discretize and advance equations (3.1) and (3.2) in time consists of a remeshed vortex method, with a penalization technique (Angot, Bruneau $\&$ Fabrie 1999) to account for the no-slip boundary condition and a projection method (Coquerelle \& Cottet 2008; Gazzola et al. 2011) to capture the action from the fluid to the body. The body geometry is represented with a characteristic function $\chi_{s}\left(\chi_{s}=1\right.$ inside the body, $\chi_{s}=0$ outside and mollified at the interface $)$ and its deformation is defined by the deformation velocity field $\boldsymbol{u}_{\text {def }}$. Further details, validation and verification of the method can be found in Gazzola et al. (2011, 2012). In this study, we discretize the domain with a uniform grid spacing of $h=L / 256$ during the optimization and $h=L / 512$ for the diagnostics reported. The mollification length of $\chi_{s}$ is set to $\epsilon=2 \sqrt{2} h$, the Lagrangian CFL condition to $L C F L=0.1$ and the penalization factor $\lambda=10^{4}$ (Gazzola et al. 2011).

\subsection{Optimizer}

Optimal shapes (characterized by $\left\{\beta_{i}^{w}\right\}_{i=1, \ldots, 4}$ and $\left\{\beta_{j}^{h}\right\}_{j=1, \ldots, 6}$ ) are identified by the stochastic optimization algorithm covariance matrix adaptation evolutionary strategy (CMA-ES) in its multi-host, rank- $\mu$ and weighted recombination form (Hansen, Muller \& Koumoutsakos 2003). CMA-ES has been shown to be robust and efficient in dealing 

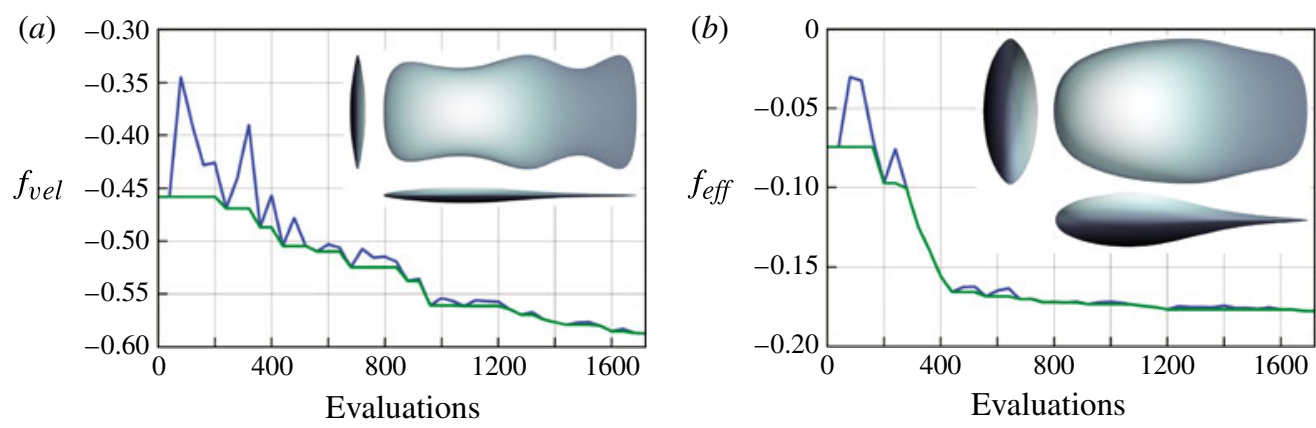

FIGURE 2. Evolution of cost functions $(a) f_{v e l}$ and $(b) f_{\text {eff }}$ against number of cost function evaluations. Blue and green lines correspond to, respectively, best solution in the current generation and best solution ever. The inset of each plot shows the corresponding optimal shape. The numerical data correspond to swimmers with $L=1, \mathscr{T}=1$ and $R e_{f i s h}=550$, simulated with resolution $h=L / 256$ due to computational costs.

with flow-related optimization problems (Kern \& Koumoutsakos 2006; Gazzola et al. 2012). Throughout this work we set the population size $p=40$, which we found to be a good compromise between robustness and computational cost. We initialize our parameters $\left(\boldsymbol{m}_{0}\right)$ to reproduce the geometry of a zebrafish larva at 5 days postfertilization (figure 1 and table 1).

The search space bounds are enforced through a rejection algorithm. We consider invalid, for numerical reasons, cases in which the vertical coordinate of $\beta_{1}^{w}$, $\beta_{N_{w}-2}^{w}, \beta_{1}^{h}$ or $\beta_{N_{h}-2}^{h}$ is smaller than the grid spacing used in our simulations. Invalid configurations are rejected by assigning a high default cost function value. Furthermore, we reject cases in which one or both profile curves cross the body midline.

\section{Results}

\subsection{Optimal shapes}

The evolution of the fitness functions, the corresponding optimal shapes and parameters are presented in figure 2 and table 1 . The width profile of the fastest swimmer is reminiscent of a thin aerofoil, with a maximum thickness of $0.03 \mathrm{~L}$ and an inflection point in the posterior half. The height profile shows a dorsal and caudal fin, connected by a caudal peduncle. The most efficient swimmer, in contrast, is thicker and blunter than the fastest swimmer, with a 4.6 times larger volume and a maximum width of $0.11 L$. An inflection point is present in the posterior width. The height profile is characterized by a large lateral area with a smooth transition between the dorsal fin and the caudal fin.

The efficiency, forward and lateral velocities of the fastest, most efficient and zebrafish larva $\left(\boldsymbol{m}_{0}\right)$ shapes are shown in figure 3. We note that, compared with the shape of the zebrafish larva, the fastest and most efficient shapes achieve a $40 \%$ higher velocity and $135 \%$ higher efficiency, respectively. These improved performances, however, come at the expense of a larger input power. In particular, the average power required by the fastest and most efficient solution during the sixth cycle is, respectively, 3.2 and 4.0 times larger than for the zebrafish larva. 


$\begin{array}{lccc} & \boldsymbol{m}_{0} & \text { Fastest } & \begin{array}{c}\text { Most } \\ \text { efficient }\end{array} \\ & & & \\ \beta_{1}^{w} & 8.9 \times 10^{-2} & 1.4 \times 10^{-2} & 7.1 \times 10^{-2} \\ \beta_{2}^{w} & 1.7 \times 10^{-2} & 4.6 \times 10^{-2} & 1.5 \times 10^{-1} \\ \beta_{3}^{w} & 1.6 \times 10^{-2} & 2.2 \times 10^{-3} & 1.3 \times 10^{-2} \\ \beta_{4}^{w} & 1.3 \times 10^{-2} & 5.8 \times 10^{-3} & 7.7 \times 10^{-3} \\ & & & \\ \beta_{1}^{h} & 5.5 \times 10^{-2} & 2.3 \times 10^{-1} & 1.6 \times 10^{-1} \\ \beta_{2}^{h} & 6.8 \times 10^{-2} & 1.7 \times 10^{-1} & 2.6 \times 10^{-1} \\ \beta_{3}^{h} & 7.6 \times 10^{-2} & 1.7 \times 10^{-1} & 2.8 \times 10^{-1} \\ \beta_{4}^{h} & 6.4 \times 10^{-2} & 2.7 \times 10^{-1} & 3.0 \times 10^{-1} \\ \beta_{5}^{h} & 7.2 \times 10^{-3} & 9.0 \times 10^{-2} & 2.3 \times 10^{-1} \\ \beta_{6}^{h} & 1.1 \times 10^{-1} & 3.0 \times 10^{-1} & 2.1 \times 10^{-1} \\ & & & \\ f_{\text {vel }} & -3.2 \times 10^{-1} & -5.0 \times 10^{-1} & -4.3 \times 10^{-1} \\ f_{\text {eff }} & -5.7 \times 10^{-2} & -8.5 \times 10^{-2} & -2.0 \times 10^{-1}\end{array}$

TABLE 1. Optimal solutions and start searching point $\boldsymbol{m}_{0}$ (approximation of zebrafish larva (Gazzola et al. 2012)). The numerical data correspond to swimmers with $L=1, \mathscr{T}=1$ and $R e_{\text {fish }}=550$.
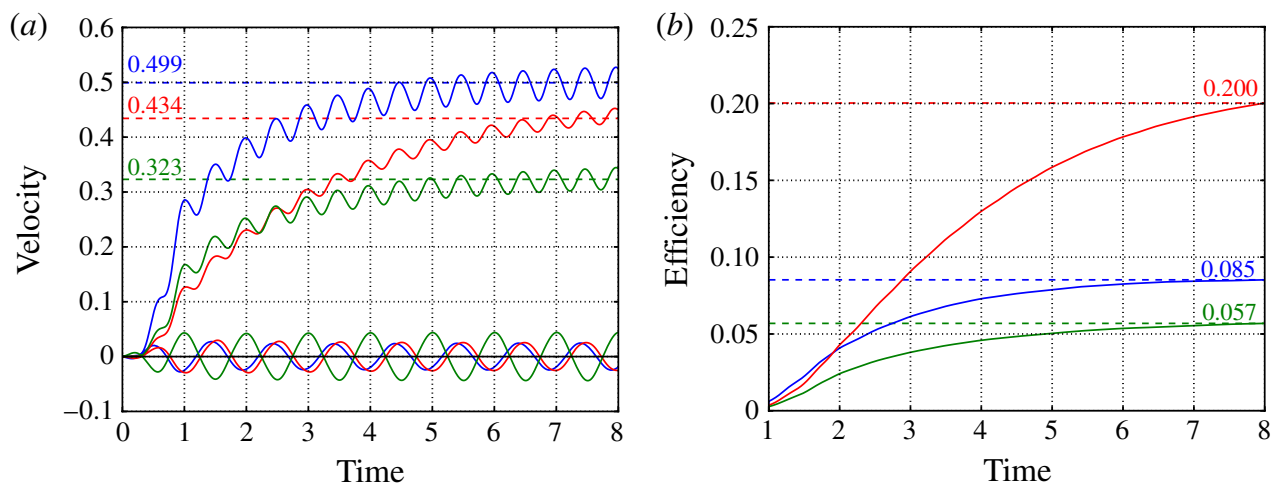

FIGURE 3. (a) Forward and lateral velocities; dashed lines denote average velocity in the eighth cycle. (b) Efficiency in a running window of length $\Delta T=\mathscr{T}$; dashed lines denote efficiency at $t=8 \mathscr{T}$. Colours denote the fastest shape (blue), the most efficient shape (red) and the zebrafish larva $\boldsymbol{m}_{0}$ (green). The numerical data correspond to swimmers with $L=1, \mathscr{T}=1$ and $R e_{\text {fish }}=550$.

\subsection{Flow analysis}

Both optimal swimmers accelerate during the first half and decelerate during the second half of each stroke. Forward and lateral velocities peak when the tail reaches its maximum lateral velocity (i.e. half-way during a stroke, after shedding the upstream 
(a)

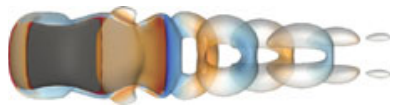

(d)

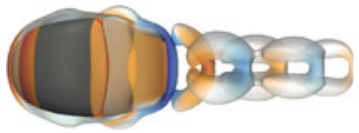

$(b)$

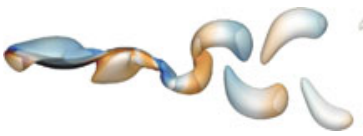

(e)

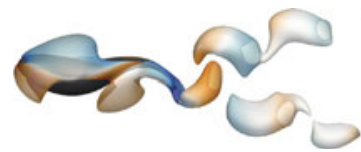

(c)

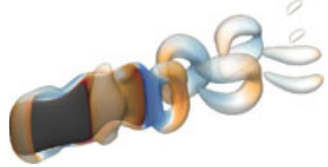

$(f)$

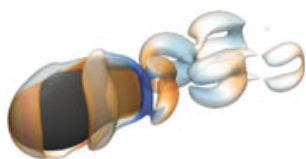

FIGURE 4. Optimal swimmers' wake structure visualized via an isosurface of the $Q$-criterion, coloured by the vertical component of the vorticity $\omega$. The swimmers are plotted in dark grey. We show $(a, d)$ side, $(b, e)$ top and $(c, f)$ perspective views for $(a-c)$ the fastest and $(d-f)$ the most efficient swimmer. Animations are presented in the supplementary material.

leg of a wake vortex ring). The minimum forward velocity is achieved at the beginning of each stroke, when the tail changes direction.

Figure 4 shows the structure of the vorticity field for the fastest swimmer visualized by the $Q$-criterion. In the second half of each tail beat, the downstream half of a lateral elliptic vortex ring is generated, which is completed in the first half of the next tail beat. Each vortex ring has a stronger circulation in the downstream half than in the upstream half. The rings are initially generated with an aspect ratio of approximately 1.9 and then relax to a circular shape. The flow field of the most efficient swimmer (figure 4) shows qualitatively the same structures as the fastest swimmer. The lack of a distinct caudal fin results in weaker upstream halves of the vortex rings, so that their asymmetric self-induced velocities cause the rings to rotate outwards around the $z$-axis. Similar to the fastest swimmer, elliptical vortex rings are generated from the tail with an aspect ratio of approximately 1.7 and relax to a circular shape downstream.

The flow-structure interaction is driven by the shape-independent midline kinematics. We estimate the vorticity associated with the midline motion as $\partial u_{n} / \partial \tau$, where $u_{n}$ is the normal velocity of the midline and $\tau$ the tangential coordinate. Visualizing this quantity reveals two midline vortices that move downstream with the travelling wave. During swimming, these midline-induced vortices modulate the boundary-layer vorticity in the midplane (figure $5 a$ ). As a result, on either side of the body stronger and weaker vortices alternate and are shed into the wake, forming a 2P vortex street (Williamson \& Roshko 1988). The three-dimensional vorticity field reveals that vortex rings are formed in the near flow around the body and travel downstream. These rings are eventually rotated by the body and shed as lateral vortex rings into the wake (figure 6).

Swimming is enabled by transferring momentum to the fluid, balancing draginduced decelerations and sustaining the average forward velocity. For anguilliform motion, the associated midline vortices modulate the vorticity on either side of the swimmer, creating coherent flow regions (figure $5 a$ ). These regions are accommodated within the concave sides of the shape and pushed downstream by the body travelling wave, accelerating the swimmer. We remark that the regions identified through this mechanism (white dashed lines in figure 5) correspond to the thrust regions visualized in figure 14 in Reid et al. (2012). The basic mechanism of propulsion through the acceleration of coherent flow regions characterizes also the C-start escape response (Gazzola et al. 2012). 
(a)

(b)

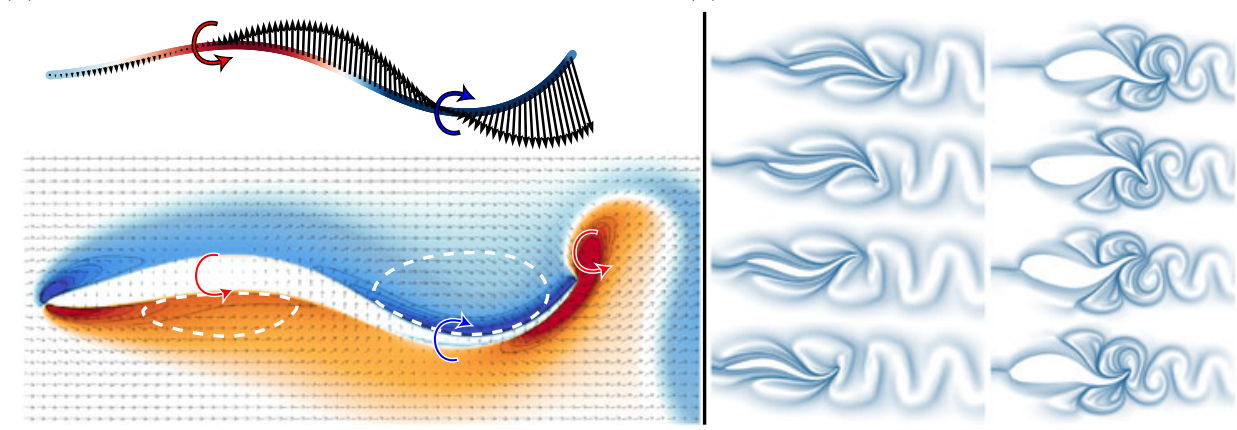

FIGURE 5. (a) Vortices generated by the midline kinematics (top) modulate the boundary layer vorticity during swimming (bottom). Black arrows denote velocity, colours denote vorticity in the midplane, white dashed lines sketch coherent flow regions. (b) The F-FTLE field, simulated over $\Delta T_{F T L E}=2 \mathscr{T}$, reveals differences in flow structures for the fastest (left) and most efficient (right) width profile in a two-dimensional simulation.

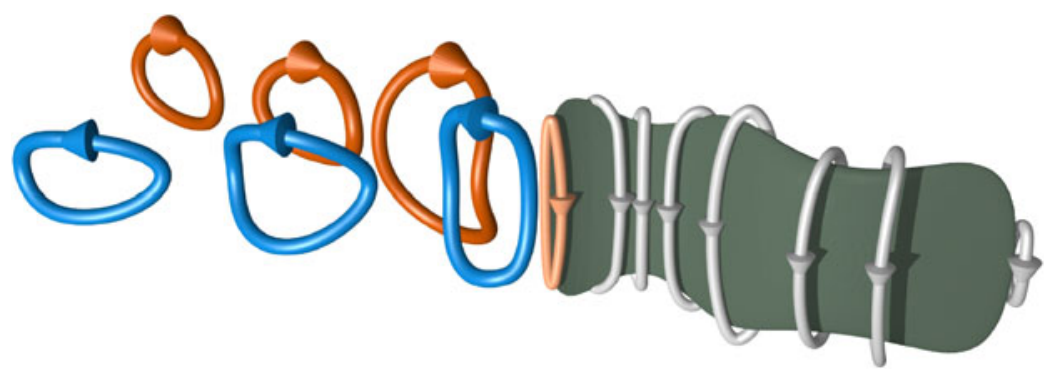

FIGURE 6. Vortex line representation of a selection of vortex rings generated by the fastest swimmer. The colours denote their orientation once they have been laterally rotated and shed into the wake.

To further elucidate this process, we simulated the optimal width profiles in two dimensions and analysed the corresponding flow field by means of the forward finite-time Lyapunov exponent field (F-FTLE), shown in figure 5(b). Two-dimensional simulations, although not able to capture the full physical problem, are instructive in understanding swimming hydrodynamics (Triantafyllou 2012). For both swimmers, the F-FTLE reveals the above-mentioned regions of coherent flow in an inertial frame of reference, which conform to the body geometry and kinematics and are shed as alternating compact vortices in the wake. Owing to the high swimming speed of the fastest swimmer, the flow regions are longitudinally stretched and extend upstream along the entire body. The most efficient swimmer, in contrast, extracts fluid from a less elongated and more circular region along the posterior $25 \%$ of the body length. This could be a characteristic of efficient swimming.

\section{Shape variations}

We systematically vary single morphological features of the obtained optimal shapes to study their impact on swimming performance. During this variation we neglect the bounding box imposed on the optimization to detect trends in an unconstrained setting. 

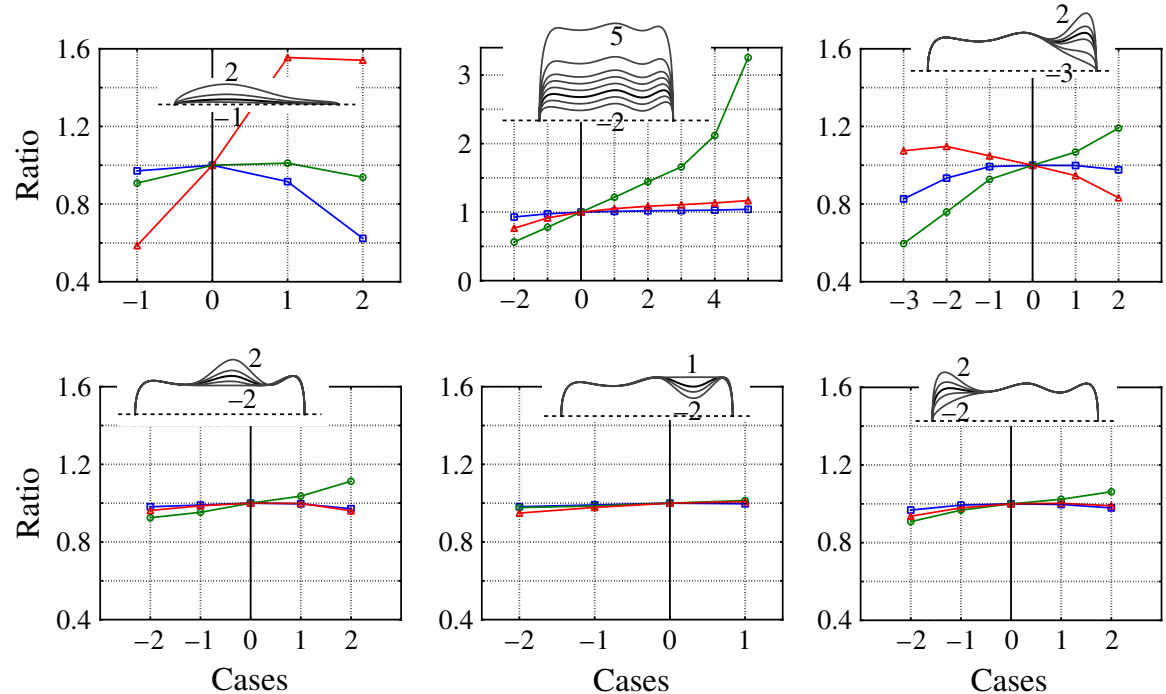

FIGURE 7. Effect of shape variations on the forward velocity (blue squares), efficiency (red triangles) and input energy (green circles) for the fast swimmer.
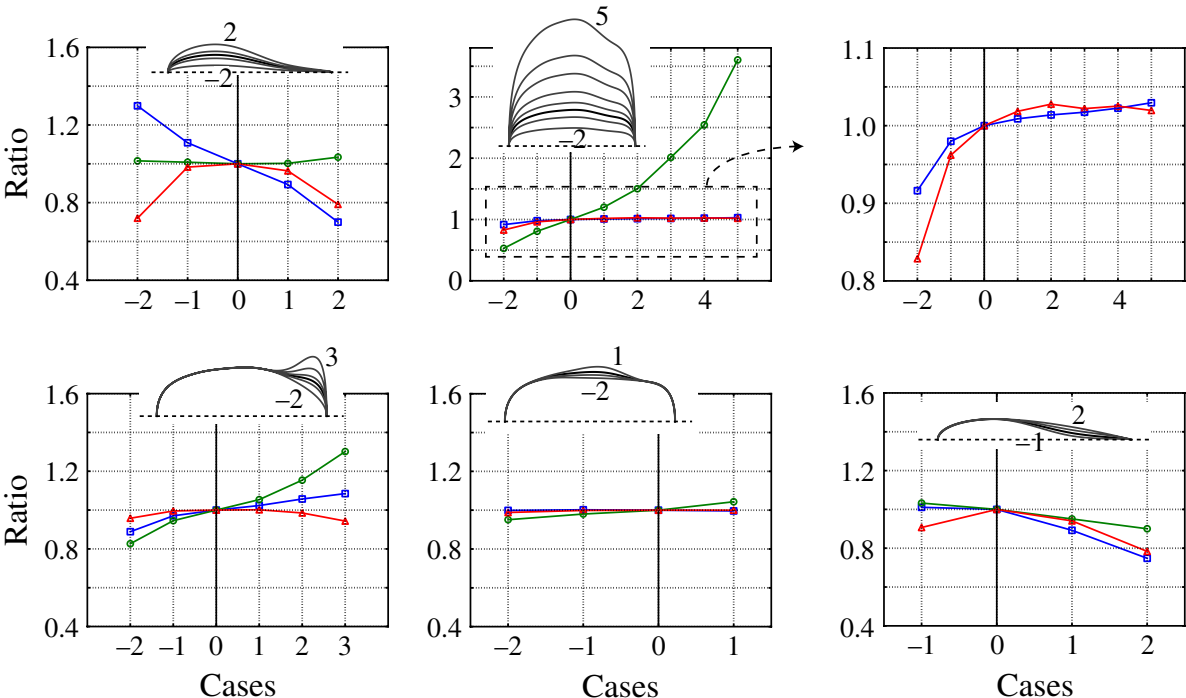

FIGURE 8. Effect of shape variations on the forward velocity (blue squares), efficiency (red triangles) and input energy (green circles) for the efficient swimmer.

Besides the forward velocity and efficiency, we show for each variation the power delivered to the fluid integrated over the sixth swimming cycle, as a measure of the associated effort, in figures 7 and 8.

\subsection{Width}

Increasing the width for the fast swimmer causes a $40 \%$ drop in forward velocity, and a dramatic improvement (up to $60 \%$ ) in efficiency. For the efficient swimmer we 


\section{W. M. van Rees, M. Gazzola and P. Koumoutsakos}

also find that thinner shapes improve speed and degrade efficiency. Thicker shapes, on the other hand, require a larger input energy than our most efficient swimmer, which degrades their efficiency.

We find that the width profile has the largest influence on the swimmers' performance. A thin shape maximizes the forward velocity by minimizing the pressure drag along the bulk of the body, whereas a thicker width profile is beneficial for efficiency by striking an optimal balance between energy losses in the wake and forward momentum of the swimmer.

\subsection{Inflection point}

The posterior part of the width profile of the optimal swimmers is characterized by an inflection point. The slenderness of the fast swimmer does not allow for a meaningful variation study, therefore we focus our analysis on the efficient case. We chose to enhance the inflection point by decreasing the value of $\beta_{3}^{w}$ (case -1 ), and to remove it by replacing the profile with a symmetric four-digit NACA aerofoil (Jacobs, Ward $\&$ Pinkerton 1933) with the same maximum thickness (case 2). In case 1 the profile is given by an average between the NACA profile and our optimal solution.

The lack of inflection point is found to be detrimental for both forward velocity $(-25 \%)$ and efficiency $(-20 \%)$. We argue, based on the F-FTLE analysis, that the presence of an inflection point increases the volume of the flow regions trapped by the body and pushed backwards during each stroke, similar to the C-start mechanism (Gazzola et al. 2012). The larger momentum transfer increases the speed, and the associated growth in useful energy improves the swimmers' efficiency.

\subsection{Caudal fin}

For the fast swimmer, the caudal fin provides a significant part of the propulsion as indicated by the drop in forward velocity when decreasing its size. The presence of a caudal fin increases the available lateral area where lateral velocities are maximal, resulting in a larger momentum transfer and therefore increased acceleration as the swimmer sweeps its tail. However, without a caudal fin the swimmer surprisingly still reaches $80 \%$ of its top speed. This shows that thrust is generated along the entire body, as coherent flow regions are pushed backwards and imparted a final acceleration by the caudal fin. Increasing the caudal fin size beyond the optimum in turn decreases the swimmer's speed. In particular, a larger caudal fin increases frictional resistance and flow separation due to a stronger adverse pressure gradient downstream of the caudal peduncle.

For the most efficient swimmer, as for the fastest one, a larger caudal fin improves the forward velocity and thereby increases its useful energy. However, the larger volume of fluid displaced requires more input energy, which dominates the growth of useful energy. Therefore swimmers with the largest caudal fins are the least efficient. This trend is also found for the fast swimmer, where the efficiency peaks at case -2 .

\subsection{Height}

We find that increasing the fast swimmer's height beyond the bounding box results in a marginal increase in forward velocity, contained within $\sim 4 \%$ of case 0 . For the efficient swimmer, case 2 (also beyond the bounding box) represents a maximum with $\sim 3 \%$ efficiency improvement over case 0 . In both cases increasing the height leads to a significant increase in the input energy required to sustain swimming, indicating that the highest shapes may not be practically feasible. These considerations justify $a$ posteriori the choice of constraining the shape optimization within a bounding box. 


\section{Optimal shapes for anguilliform swimmers at intermediate Re numbers}

\subsection{Head profile, dorsal fin and caudal peduncle}

Naturally occurring anguilliform swimmers are often characterized by a torpedo-like head profile, pronounced dorsal fin and/or caudal peduncle. Varying these features in our optimal shapes leads to negligible performance degradation (at most $\sim 3 \%$ ) for both speed and efficiency. Therefore, the widespread appearance of these features is not justified by speed or efficiency alone. Such traits may improve manoeuvrability or serve biological constraints.

\section{Conclusions}

We have presented a reverse engineering process to obtain the fastest and most efficient shapes for self-propelled anguilliform swimmers at moderate Reynolds numbers. This optimization relies on coupling an evolution strategy with threedimensional flow simulations using remeshed vortex methods.

Flow analysis reveals that the midline kinematics drives the formation of coherent flow regions along either side of the swimmer, which are pushed downstream by the body travelling wave. Through this mechanism the swimmer transfers momentum to the flow and, consequently, accelerates.

The optimal shapes possess distinct features that are also widespread across natural swimmers, such as a streamlined profile, dorsal fin, caudal fin and caudal peduncle. However, the overall appearance of the optimal morphologies differs from natural anguilliform swimmers. The obtained optimal shapes outperform the larval zebrafish geometry by $40 \%$ and $135 \%$ for speed and efficiency, respectively. We note that the optimal solutions require large input power (3-4 times more than a zebrafish larva), which may not be attainable by natural swimmers due to muscular or metabolic limitations, but might be achievable by robotic devices. The most relevant morphological features identified are the swimmers' thickness, inflection point and caudal fin. Interestingly, the removal of the caudal fin from the fastest shape still allows it to reach $80 \%$ of its maximum speed, showing how anguilliform swimming generates thrust along the entire body. Furthermore, based on flow analysis, we argue that the presence of an inflection point allows an increased momentum transfer, improving speed $(+25 \%)$ and efficiency $(+20 \%)$.

This study indicates that engineered swimmers have the potential to outperform natural anguilliform swimmers in speed and efficiency, for the flow regime considered herein.

\section{Supplementary material}

Supplementary material and movies are available at http://dx.doi.org/10.1017/jfm. 2013.157.

\section{References}

Angot, P., Bruneau, C. H. \& Fabrie, P. 1999 A penalization method to take into account obstacles in incompressible viscous flows. Numer. Math. 81 (4), 497-520.

Bartol, I. K., Gharib, M., Webb, P. W., Weihs, D. \& Gordon, M. S. 2005 Body-induced vortical flows: a common mechanism for self-corrective trimming control in boxfishes. J. Expl Biol. 208 (2), 327-344.

BorAZJANi, I. \& Sotiropoulos, F. 2010 On the role of form and kinematics on the hydrodynamics of self-propelled body/caudal fin swimming. J. Expl Biol. 213 (1), 89-107. 


\section{W. M. van Rees, M. Gazzola and P. Koumoutsakos}

Carling, J., Williams, T. L. \& Bowtell, G. 1998 Self-propelled anguilliform swimming: simultaneous solution of the two-dimensional Navier-Stokes equations and Newton's laws of motion. J. Expl Biol. 201 (23), 3143-3166.

Coquerelle, M. \& Cottet, G. H. 2008 A vortex level set method for the two-way coupling of an incompressible fluid with colliding rigid bodies. J. Comput. Phys. 227 (21), 9121-9137.

Eloy, C. 2013 On the best design for undulatory swimming. J. Fluid Mech. 717, 48-89.

Gazzola, M., Chatelain, P., van Rees, W. M. \& Koumoutsakos, P. 2011 Simulations of single and multiple swimmers with non-divergence free deforming geometries. J. Comput. Phys. 230 (19), 7093-7114.

Gazzola, M., van Rees, W. M. \& Koumoutsakos, P. 2012 C-start: optimal start of larval fish. J. Fluid Mech. 698, 5-18.

GraY, J. 1933 Studies in animal locomotion I. The movement of fish with special reference to the eel. J. Expl Biol. 10 (1), 88-104.

Hansen, N., Muller, S. D. \& Koumoutsakos, P. 2003 Reducing the time complexity of the derandomized evolution strategy with covariance matrix adaptation (CMA-ES). Evol. Comput. 11 (1), 1-18.

HeRTEl, H. 1966 Structure, Form, Movement. Reinhold.

JACOBS, E. N., WARD, K. E. \& PInKerTOn, R. M. 1933 The characteristics of 78 related airfoil sections from tests in the variable-density wind tunnel. NACA Tech. Rep. 460.

Kern, S. \& Koumoutsakos, P. 2006 Simulations of optimized anguilliform swimming. J. Expl Biol. 209 (24), 4841-4857.

Muller, U. K., van den Boogaart, J. G. M. \& van Leeuwen, J. L. 2008 Flow patterns of larval fish: undulatory swimming in the intermediate flow regime. J. Expl Biol. 211 (2), 196-205.

Nachtigall, W. \& Bluechel, K. 2000 Das Grosse Buch der Bionik. Deutsche Verlags-Anstalt.

Nawroth, J. C., Lee, H., Feinberg, A. W., Ripplinger, C. M., McCain, M. L., Grosberg, A., DABIRI, J. O. \& PARKER, K. K. 2012 A tissue-engineered jellyfish with biomimetic propulsion. Nat. Biotech. 30 (8), 792-797.

Reid, D. A. P., Hildenbrandt, H., Padding, J. T. \& Hemelrijk, C. K. 2012 Fluid dynamics of moving fish in a two-dimensional multiparticle collision dynamics model. Phys. Rev. E 85, 021901.

TOKIC, G. \& YUE, D. K. P. 2012 Optimal shape and motion of undulatory swimming organisms. Proc. R. Soc. B: Biol. Sci. 279 (1740), 3065-3074.

Triantafyllou, M. S. 2012 Survival hydrodynamics. J. Fluid Mech. 698, 1-4.

Triantafyllou, M. S. \& Triantafyllou, G. S. 1995 An efficient swimming machine. Sci. Am. 272 (3), 64-70.

Tytell, E. D. 2004 The hydrodynamics of eel swimming - II. Effect of swimming speed. J. Expl Biol. 207 (19), 3265-3279.

Wilga, C. D. \& LAUder, G. V. 2004 Biomechanics: hydrodynamic function of the shark's tail. Nature 430 (7002), 850.

Williamson, C. H. K. \& RoshKo, A. 1988 Vortex formation in the wake of an oscillating cylinder. J. Fluids Struct. 2, 355-381. 